\title{
HYPOSPADIAS IN A SHELTIE PUPPY: A CASE REPORT
}

\author{
T. KUZNETSOVA, A. FEDULOV, E. FEDULOVA, \\ B. SEMENOV \& A. PRUSAKOV
}

\author{
St. Petersburg State Academy of Veterinary Medicine, St. Petersburg, \\ Russian Federation
}

\section{Summary}

Kuznetsova, T., A. Fedulov, E. Fedulova, B. Semenov \& A. Prusakov, 2020. Hypospadias in a Sheltie puppy: A case report. Bulg. J. Vet. Med., 23, No 4, 509-516.

Hypospadias in dogs is a rare pathology in the veterinary practice. The manifestation of hypospadias in dogs is diverse, since there is a varying degree of damage to the urogenital apparatus. The owners of a Sheltie puppy at the age of 3 days came to the clinic due to the difficulty of determining sex, the presence of inflammation of the anus and abdominal skin, defecation and urination violations. Clinical examination of the puppy showed a blind-closed preputial sac, absence of the ventral wall of the prepuce and an open urogenital urine trough was located in its place in the abdominal wall area. On examination of the puppy at the age of 28 days, hyperaemia and swelling of the anus were noted, as well as prolapse of the rectum. Findings of the examination at the age of 4 months consisted of drying of the mucous part of the open urogenital canal chute and accumulation of pus in the underdeveloped preputial sac. Bilateral cryptorchidism and the absence of the scrotum were also found out. A decision on the surgical treatment was made. The anus and the opening of the urethra were separated to form a urethrostomy in the scrotum and restore the integrity of the anus. On the $5^{\text {th }}$ post operative day, oedema and stricture of the reconstructed urethra resulted in difficulty urinating, followed by the formation of urinary fistula in the perineal region below the anus opening. As a result of the chosen surgical treatment approach, the problem with contact dermatitis of the perineum and pollakiuria was solved.

Key words: congenital hypospadias, dog, operation

Hypospadias in dogs is a rare pathology in the veterinary practice. In each case, animals have different degrees of the urogenital organs' damage. Currently, there is no unambiguous scheme of surgical treatment of this pathology. Each case of hypospadias is individual and requires a creative approach for surgical treatment from the practicing veterinarian. With this regard, data on the possible methods of surgical correction of this pathology are of great practical value. Considering this, we found it necessary to describe the present case hypospadias and the method of its surgical treatment. 
Hypospadias is a congenital defect of the male genital organs, caused by improper fusion of urogenital folds during the foetal period. With this pathology, the prepuce is often underdeveloped or completely absent (Rübben \& Stein, 2017). The location of the hypospadias varies: the opening of the urethra can be located a few centimeters from the end of the penis (glandular hypospadias), in the region of the long part of the glans penis (balanic hypospadias), in the scrotum (scrotal hypospadias), in the perineal region (perineal hypospadias. Local dermatitis may develop due to irritation with urine in these animals. Difficult urination may also occur. The severity of the clinical manifestations of hypospadias depends on the location of the opening of the urethra. The severity is higher if the opening is more caudal. (Grossman \& Baltzer, 2012; Meyers-Wallen, 2012).

Hypospadias is often accompanied by such congenital malformations as cryptorchidism, persistent penile frenulum, penis or prepuce underdevelopment, hermaphroditism, pseudohermaphroditism (Switonski et al., 2012).

The main causes of hypospadias are genetic disorders and hormonal changes, associated with low testosterone levels in an organism (Severgina et al., 2016). Moreover, this pathology is characterised by high genetic heterogeneity, which also makes some difficulties to ascertain the causes of its occurrence (Cassata et al., 2008).

It is known that using exogenous progesterone or estrogen during pregnancy can cause the development of hypospadias in foetuses (Rübben \& Stein, 2017).

Experimental studies on mice and rats have shown the destructive effect on the male reproductive system of such chemicals as flutamide (Sinclair et al., 2017) and dibutyl phthalate. Prenatal exposure to these drugs led to hypospadias, cryptorchidism, or other congenital malformations in males. (Zhang \& Liu, 2018).

The literature also describes cases of hypospadias in males of other animal species - bull (Mihsler et al., 2015), stallion (Brink \& Schumacher 2011; Harrison \& Mushtaq, 2017), cat (King \& Johnson, 2000), mouflon (Beregi et al., 2013).

It is known that in German shepherds (Switonski et al., 2018) and Boston terriers hypospadias are more common than in other dog breeds (Grossman \& Baltzer, 2012). Abnormal development of the urethra in dogs is associated with impaired embryogenesis. As a consequence of the displacement of the genital tubercle caudally during embryogenesis, a disturbance of the mesodermal lamina occurs. The consequence of this violation is the nonclosure of the anterior wall of the urogenital canal. In this way, a urogenital groove is formed instead. The epithelium lining the groove is dried out after birth. The epithelium dies away under the influence of external factors. At the same time, it is possible to observe ulceration of the mucous membrane of the groove, as well as its changes associated with the impact of a secondary infection. The absence of an epithelial lining on the mucous membrane of the urogenital groove should be considered when stitching its edges. This manipulation is impossible without the bougienage of a newly created channel. In this case, due to the lack of epithelial lining, the mucous membrane is prone to the formation of adhesive inflammation. (Cashmore \& Ladlow, 2016).

\section{Case presentation}

The owners of a 3-day Sheltie puppy contacted the clinic due to difficulties with sex determination because of atypic exter- 
nal genital organs. There was an inflammation in the anus and abdominal skin, violation of acts of defecation and urination. Also, urine was excreted in the puppy through the anus.

The physical examination of a puppy at the age of 3 days showed a blind-closed preputial cavity at the background of good general clinical condition. The absence of the ventral part of the prepuce was also observed. In its place in the field of the abdominal wall, there was a unclosed urinary groove of the urogenital canal, located from the preputial cavity to the anus.

On physical examination of a puppy at the age of 28 days, hyperaemia and swelling of the anus, prolapse of the caudal part of the rectum were noted (Fig. 1). On the ventral part of the anus, a urinary fistula was diagnosed, which opened at the border of the mucous membrane of the anus and rectum. In this area, the circular integrity of the anus was disrupted by $1 / 3$. Thus, when urinating, the urine was in contact with the anus, and during the act of defecation, the faeces were in contact with the mucous membrane of the urinary fistula. Because of these effects, inflammation of the mucous membranes of the anus and urinary fistula was observed.

In order to assess the growth of the puppy, live body weight gain was simultaneously recorded and compared with that of the second puppy (female) without pathology from the litter. Puppies received feed with the same qualitaty and quantity and were kept in the same conditions. The live weight of puppies at birth was approximately the same: $199 \mathrm{~g}$ in the puppy with hypospadias and $228 \mathrm{~g}$ in the healthy puppy. On the $28^{\text {th }}$ day of life, the live weight of the puppy with hypospadias was by $305 \mathrm{~g}$ less compared to the healthy one.

In connection with the satisfactory general condition of the puppy and the positive tendency of its growth, it was decided to postpone surgery until the age of 4 months. The body weight gained at this age would allow adequate general anaesthesia.

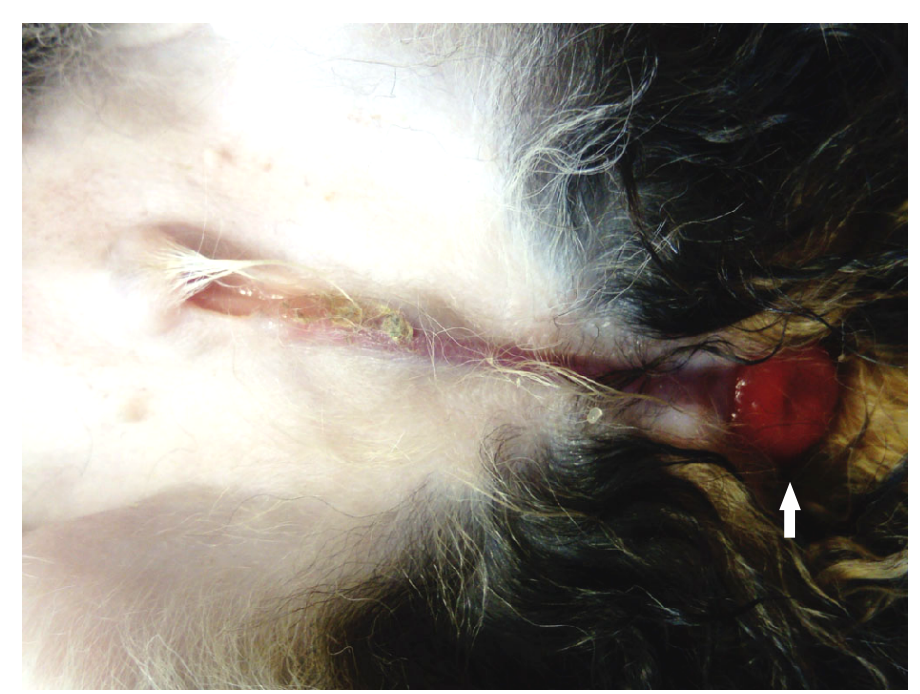

Fig. 1. View of hypospadias of the Sheltie puppy at the age of 28 days: the arrow shows prolapse of the caudal part of the rectum. 


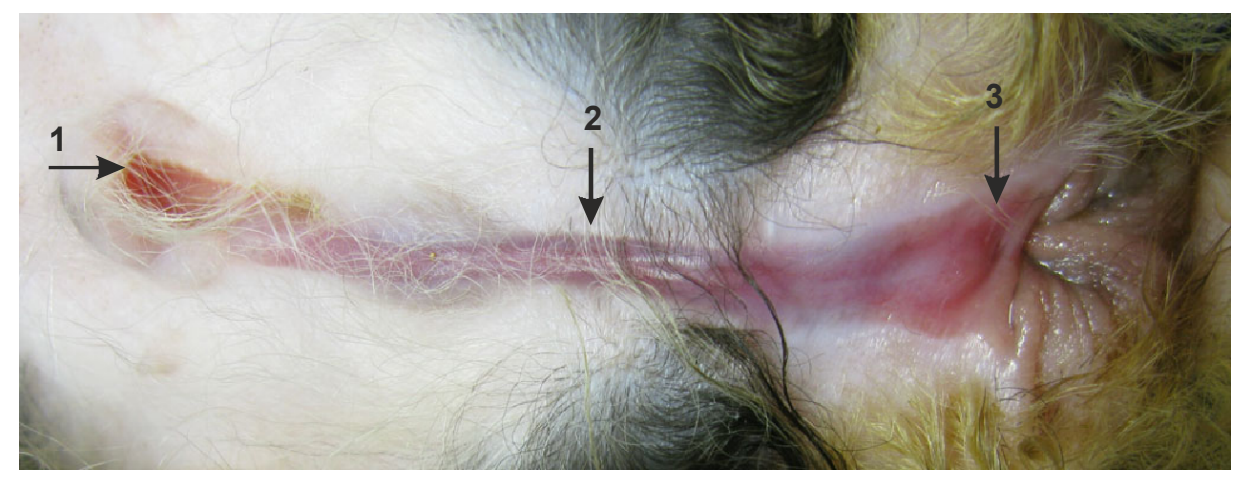

Fig. 2. Sheltie puppy's perineum area with hypospadias at the age of 4 months: 1 - accumulation of pus in the underdeveloped preputial cavity; 2 - dryness of the mucous part of the not closed groove of the urogenital canal; 3 - swelling and hyperaemia of the anus and urinary fistula.

On physical examination of the animal at 4 months of age, dryness of the mucous part of the unclosed groove of the urogenital canal, accumulation of pus in the underdeveloped preputial cavity and initial stage of tissue necrosis were noted (Fig. 2). Dermatitis of the perineum skin due to constant irritation of the urine, sticking of hair to the mucous membrane of the urethra was found. During the palpation, undescended testes were found in the groin area in the absence of the scrotum.

This pathological condition of the dog required the owners to take care of the crotch area and wash the coat due to the constant urine entering the medial surface of the hip. The animal was anxious at the time of urination, defecation, including pollakiuria.

After taking history and on the basis of the results from the clinical examination of the puppy at the age of 4 months, we decided to perform a surgical operation. The surgical team identified the following goals of the upcoming operation: first, preparation of the mucous membrane of the urethra from the mucous membrane of the anus and rectum with the restoration of the circular integrity of the anus due to the tissues of the skin of the anus; second, formation of an urethrostomy cranial to the fistulous opening with the help of reconstruction and plastics of the urethra; and third, removal of an underdeveloped penis and excision of the preputial cavity.

Operational intervention for the removal of undescended testes was postponed until puberty.

The operation was performed in the clinic for small animals. The puppy was fixed in the dorsal position on the surgery table. Surgery manipulations were done under general anaesthesia. For premedication, xylazine 2\% (Xylazine, Netherlands) at a dose of $0.1 \mathrm{~mL} / \mathrm{kg}$ was used, and propofol $10 \%$ (Propofol, Austria) at the rate of $5 \mathrm{mg} / \mathrm{kg}$ was used for induction of anaesthesia.

A lanceolate incision was used to remove the underdeveloped preputial cavity, the vessels were ligated. After isolating and ligating the dorsal arteries and veins of the penis with a circular incision, the bone and the bulb of the glans penis were dissected (Fig. 3). Knotted stitches were laid in the transverse direction on the formed stump of the cavernous bodies. In 
the place of the removed prepuce, knotted sutures from the navel to the stump of the penis were laid on the skin along the midline.

Through the opening of the urinary fistula, a Foley catheter No. 5 (Russia) was inserted in the line of the bladder. After that, two parallel incisions were performed to excise the edges of the unclosed urethral mucosa and mobilise the edges over the catheter with suturing the mucosa with resorbable suture material PGA 4/0 (Russia). In the area of the anus defect after separation of the mucous membrane of the urethra, adjacent sutures were applied with the same suture material. On the skin above the urethra, simple knotted sutures with Dacron 2/0 (Russia) were applied. The ventral part of the mucous membrane of the urethra was sewn to the skin with the formation of urethrostomy in the area of the proposed localisation of the scrotum. Fig. 4 and 5 present some steps of the operation.

Post operative treatment included systemic antibiotic therapy with $50 \mathrm{mg} / \mathrm{kg}$ ceftriaxone (Ceftriaxone, Russia) once daily for 7 days and wound dressing with chlorhexidine (Chlorhexidine, Russia) and fucorcin (Fucorcin, Russia).

The physical examination of the puppy on the $5^{\text {th }}$ post operative day showed difficulty in urination due to oedema and stricture of the reconstructed urethra. A urinary fistula in the perineal region was formed for $1 \mathrm{~cm}$ below the operatively formed circular opening of the anus.

Sutures were removed on day 14 . On examination of the animal, the general condition was satisfactory. There was a fusion of urethrostomy, and urination passed through the urinary fistula.

As a result of surgery, the animal did not experience discomfort during urination and defecation. During urination uniformity of the jet was observed: the urine did not fall on the skin of the medial surface of the pelvic limbs. Hyperaemia and swelling of the anus were absent. Dermatitis of the skin in the perineum, anus and urinary fistula was not observed, nor any uraemic smell of dog's haircoat.

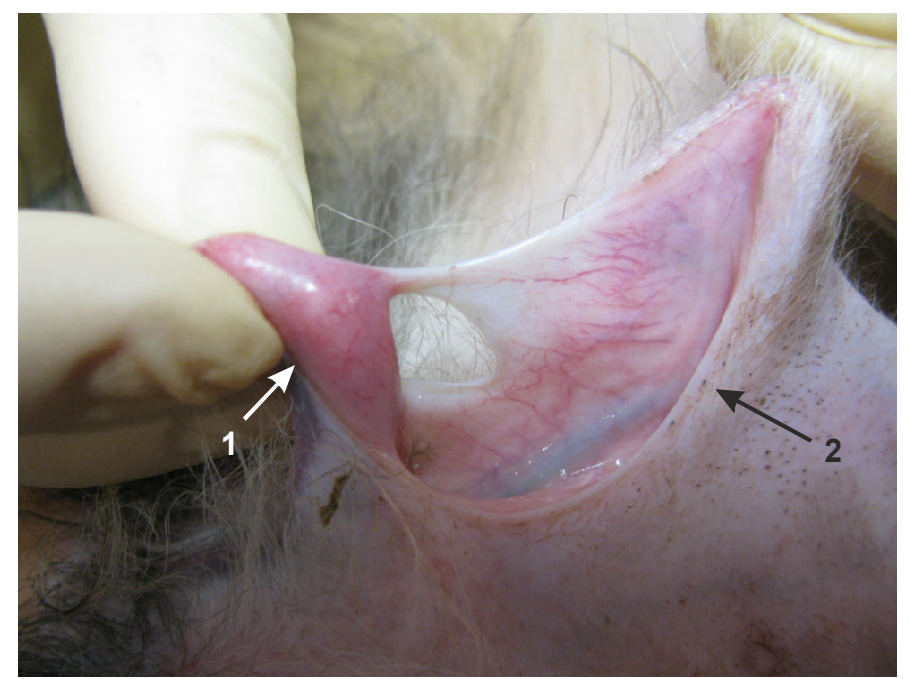

Fig. 3. Topography of the preputial cavity and penis in the Sheltie puppy at the age of 4 months: 1 - an underdeveloped penis; 2 - preputial cavity. 


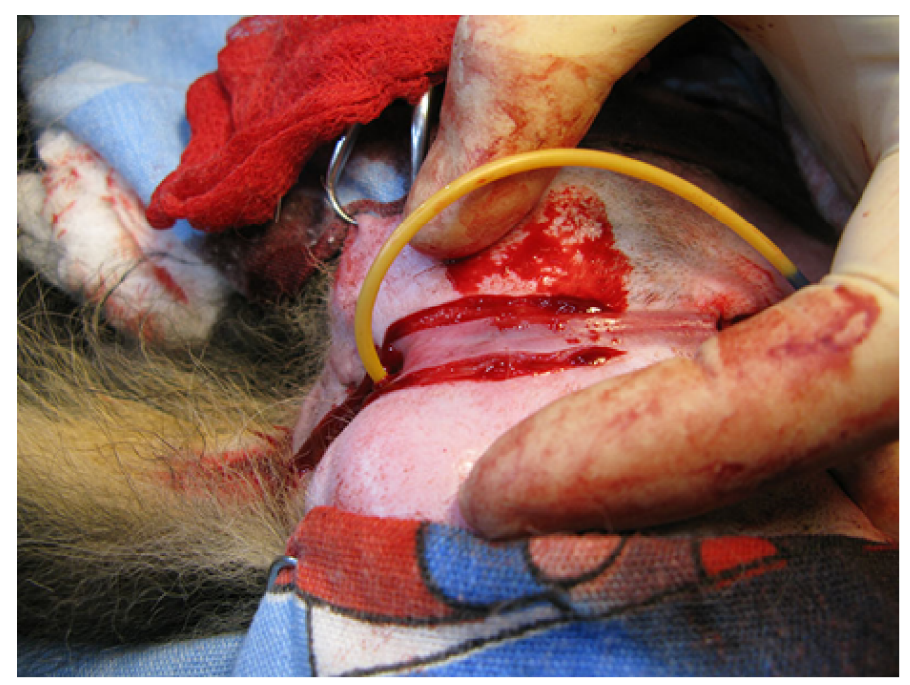

Fig. 4. Mobilisation of the edges of the urogenital groove of the Sheltie puppy with hypospadias.

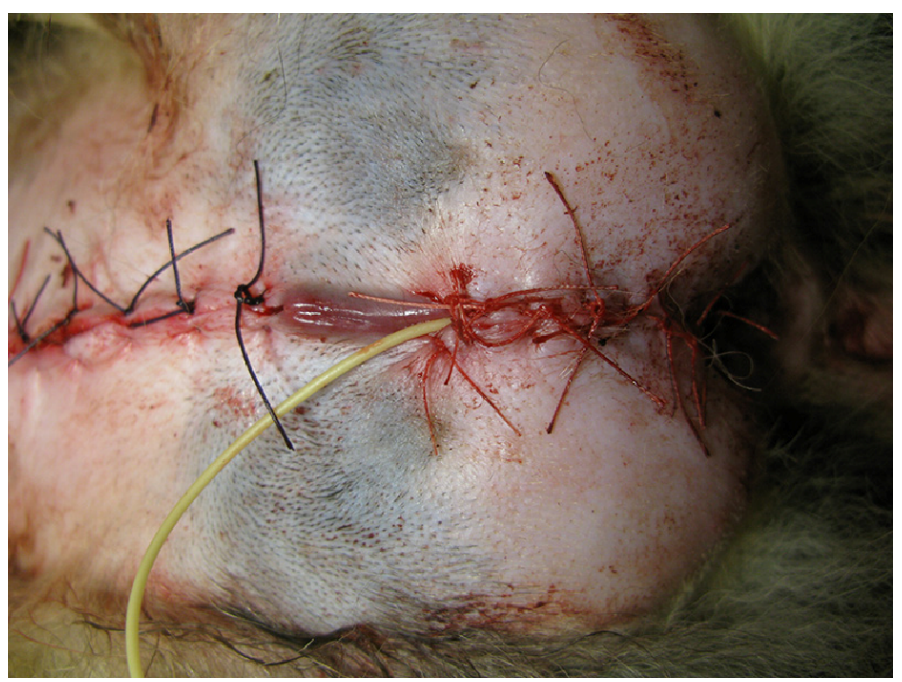

Fig. 5. Formed urethrostomy at the final stage of the operation.

This dog was monitored for 1.5 years, both in terms of physical development and in terms of behavioural reactions. The animal behaved actively, showed an adequate interest in food. After the operation, no pathological conditions and diseases have been diagnosed. After walking the dog did not require additional hygienic measures. Live body weight of the dog meets the breed standard, the animal is clinically healthy.

The choice of treatment depends on the severity and localisation of the defect. In mild cases of hypospadias, surgical treatment is usually not required, patients are recommended to have more frequent 
bathing and the use of hydrophobic ointments in the perineal area (Hedlund, 2002). Surgical treatment is used in more severe cases of hypospadias. The extent of surgery also depends on the severity of this pathology. With minor defects, a persistent frenulum can be incised, the penis can be shortened, and its amputation with the suture of a too wide opening of the prepuce (Galanty et al., 2008, Guimarães et al., 2013). In some cases, the reconstruction of the prepuce is practiced (Ndikuwera, 2005; Grossman \& Baltzer, 2012). More complex cases of hypospadias require serious surgical procedures. In these cases, various approaches are possible to remove the testes, scrotum, penis, and the formation of urethrostomy (Galanty et al., 2008; Adelsberger \& Smeak, 2009).

As a result of surgical treatment of the hypospadias in the puppy, the anus and urethra were separated, forming a urethrostomy in the scrotum and restoring the integrity of the anus. This manipulation solved the problem of contact dermatitis of the perineum and pollakiuria. Each case of hypospadias, as anomalies of the urogenital tract, is unique and further research of this pathology is necessary.

\section{REFERENCES}

Adelsberger, M. E. \& D. Smeak, 2009. Repair of extensive perineal hypospadias in a Boston terrier using tubularized incised plate urethroplasty. Canadian Veterinary Journal, 50, 937-942.

Beregi, A., K. Erdélyi, K. Fodor, L. Molnar \& S. Csányi, 2013. First description of hypospadias and inguinal bilateral cryptorchidism in mouflon (Ovis Gmelini Musimon) - short communication. Acta Veterinaria Hungarica, 61, 473-476.
Brink, P. \& J. Schumacher, 2011. Hypospadias in a Friesian gelding. Veterinary Surgery, 40, 120-123.

Cashmore, R. \& J. Ladlow, 2010. Creation of a urethral conduit from a preputial indirect flap in a dog with perineal hypospadias. Veterinary Surgery, 39, 14-20.

Cassata, R., A. Iannuzzi, P. Parma, L. De Lorenzi, V. Peretti, A. Perucatti, L. Iannuzzi \& G. Di Meo, 2008. Clinical, cytogenetic and molecular evaluation in a dog with bilateral cryptorchidism and hypospadias. Cytogenetic And Genome Research, 120, 140-143.

Galanty, M., P. Jurka \& P. Zielińska, 2008. Surgical treatment of hypospadias. Techniques and results in six dogs. Polish Journal of Veterinary Sciences, 11, 235 243.

Grossman, J. \& W. Baltzer, 2012. Use of a preputial circumferential mucosal flap for hypospadias management in a Boston terrier. Journal of Small Animal Practice, 53, 292-296.

Guimarães, L. D., E. Bourguignon, L. C. Santos, T. S. Duarte, E. C. Andrade \& A. P. B. Borges, 2013. Canine perineal hypospadias. Arquivo Brasileiro de Medicina Veterinaria e Zootecnia, 65, 1647-1650.

Harrison, J. \& I. Mushtaq, 2017. Single stage urethroplasty for perineal hypospadias in a horse: A case report. Journal of Pediatric Urology, 13, 84-85.

Hedlund, C. S., 2002. Surgery of the male reproductive tract. In: Small Animal Surgery, $2^{\text {nd }}$ edn, Mosby, St. Louis, pp. 662 665.

King, G. \& E. Johnson, 2000. Hypospadias in a Himalayan cat. Journal of Small Animal Practice, 41, 508-510.

Meyers-Wallen, V. 2012. Gonadal and sex differentiation abnormalities of dogs and cats. Sexual development: Genetics, Molecular Biology, Evolution, Endocrinology, Embryology, and Pathology of Sex Determination and Differentiation, 6, 46-60. 
Mihsler, L., H. Hussein \& A. Wehrend, 2015. Perineal hypospadia in a red Holstein-calf. A case report. Tierärztliche Praxis. Ausgabe G, Grosstiere/Nutztiere, 43, 105108.

Ndikuwera, J., 2005. A case of hypospadias in a dog. Irish Veterinary Journal, 58, 504506 .

Rübben, I. \& R. Stein, 2017. Hypospadias: Insights and challenges. Der Urologe A, 56, 1256-1265.

Severgina, L. O., L. B. Menovshchikova \& I. A. Korovin, 2016. Modern view on the development and treatment of hypospadias. Russian Bulletin, VI, 50-56 (RU).

Sinclair A., M. Cao, A. Pask, L. Baskin \& G. Cunha, 2017. Flutamide-induced hypospadias in rats: A critical assessment. Differentiation, 94, 37-57.

Switonski, M., R. Payan-Carreira, M. Bartz, J. Nowacka-Woszuk, I. Szczerbal, B. Colaço, M. Pires, M. Ochota \& W. Nizanski, 2012. Hypospadias in a male $(78, X Y$; SRY-positive) dog and sex reversal female (78,XX; SRY-negative) dogs: Clinical, histological and genetic studies. Sexual Development: Genetics, Molecular Biology, Evolution, Endocrinology, Embryology, and Pathology of Sex Determination and Differentiation, 6, 128-134.
Switonski, M., S. Dzimira, R. Aleksiewicz, I. Szczerbal, J. Nowacka-Woszuk, P. Krzeminska, T. Deska \& W. Nizanski, 2018. Hypospadias is not rare in dogs: Five new cases, a retrospective study, and a review of the literature. Sexual Development: Genetics, Molecular Biology, Evolution, Endocrinology, Embryology, and Pathology of Sex Determination And Differentiation, doi: 10.1159/000490079.

Zhang, Z. \& W. Liu, 2018. Role of PERKeIF2 $\alpha$ signaling pathway in fetal male rats with hypospadias induced by di-n-butyl phthalate. The Kaohsiung Journal of Medical Sciences, 34, 487-493.

Paper received 29.12.2018; accepted for publication 22.02.2019

\section{Correspondence:}

Tatyana Kuznetsova

Faculty of Veterinary Medicine, St. Petersburg State Academy of Veterinary Medicine, Russia tel: +79500288547

e-mail: kuznett@yandex.ru 